\title{
miR-367 promotes tumor growth by inhibiting FBXW7 in NSCLC
}

\author{
GUODONG XIAO $^{1}$, XIAO GAO $^{1}$, XIN SUN $^{1}$, CHENGCHENG YANG $^{2}$, BOXIANG ZHANG $^{1}$, \\ RUIYING SUN ${ }^{3}$, GUANGHONG HUANG ${ }^{1}$, XIANG LI $^{1}$, JIAN LIU ${ }^{1}$, NING DU ${ }^{1}$, \\ DAPENG LIU ${ }^{1}$, RUI LIANG ${ }^{4}$, HONG REN ${ }^{1}$ and SIDA QIN ${ }^{1}$ \\ ${ }^{1}$ Second Department of Thoracic Surgery and ${ }^{2}$ Department of Oncology, The First Affiliated Hospital of \\ Xi'an Jiaotong University; ${ }^{3}$ Department of Respiratory Medicine, The Second Affiliated Hospital of \\ Xi'an Jiaotong University, Xi'an, Shaanxi; ${ }^{4}$ Department of Hepatobiliary Chest Surgery, \\ Shaanxi Provincial Corps Hospital of Chinese People's Armed Police Force, Taiyuan, Shanxi, P.R. China
}

Received November 17, 2016; Accepted June 12, 2017

DOI: $10.3892 /$ or.2017.5755

\begin{abstract}
R-367 is one of the most abundant miRNAs in human embryonic stem cells (hESCs) and is mainly involved in maintaining the pluripotency of stem cells. However, its role in cancer development remains poorly understood. In the present study, we explored the function and mechanism of the endogenous miR-367 in non-small cell lung cancer (NSCLC). In the present study, we demonstrated that the level of miR-367 in NSCLC was significantly higher than that in adjacent normal tissues, and its upregulation was positively correlated with tumor size, tumor differentiation and tumor-node-metastasis (TNM) stage. miR-367 was an indicator of a poorer prognosis in NSCLC patients. Furthermore, overexpression of miR-367 significantly inhibited apoptosis and enhanced proliferation by promoting cell cycle transition from $\mathrm{G} 1$ to $\mathrm{S}$ phase. In contrast, knockdown of miR-367 markedly reversed the cellular events observed with miR-367 overexpression. Moreover, we identified that F-box and WD repeat domain-containing 7 (FBXW7) is a novel target of miR-367. It reverses the oncogenic effects of miR-367 by downregulating its substrates, c-Myc and c-Jun, in NSCLC cells. Finally, studies in vivo revealed that knockdown of miR-367 inhibited the growth of xenografts in the nude mice by increasing the expression of FBXW7. In summary, our findings indicate that miR-367 exerts tumorpromoting effect by negatively regulating FBXW7 in NSCLC, and it could become a potential therapeutic target for NSCLC intervention.
\end{abstract}

Correspondence to: Professor Hong Ren or Dr Sida Qin, Second Department of Thoracic Surgery, The First Affiliated Hospital of Medical College, Xi'an Jiaotong University, 277 Yanta West Road, Xi'an, Shaanxi 710061, P.R. China

E-mail: renhong@xjtu.edu.cn

E-mail: sida.qin@yahoo.com

Key words: miR-367, FBXW7, NSCLC, tumor growth

\section{Introduction}

Lung cancer is the leading cause of cancer-related death worldwide, and non-small cell lung cancer (NSCLC) accounts for $80 \%$ of all lung cancer cases (1). Although huge progress has been achieved in the diagnosis and treatment of NSCLC, more than $70 \%$ of the patients lose the opportunity of surgery due to advanced tumor stage. In addition, the 5-year survival rate for NSCLC patients with this stage is less than $15 \%$ (2). Therefore, it is important to explore specific biomarkers for the diagnosis of early stage NSCLC. As a group of endogenous non-coding small RNAs (18-23 nt), microRNAs (miRNAs) are known to regulate the translation of mRNAs as target genes through directly binding to their 3 '-untranslated region (3'-UTR). Many studies have shown that dysfunction of miRNAs promote the development of a variety of malignant tumors, including lung cancer.

miR-367, an miRNA located on chromosome 4 q25, belongs to the miRNA-302/367 cluster (3). Previous studies have mainly investigated the functions of miR-367 in embryonic stem cell self-renewal and the maintain of pluripotency (4). Recent studies revealed that miR-367 participates in the tumorigenesis of various types of cancers, such as hepatocellular carcinoma (5), medulloblastoma (6) and pancreatic cancer (7). In contrast, in the study of gastric carcinoma, miR-367 inhibited cell migration and invasion, and its expression in cancer tissues was much lower than that in normal stomach tissues (8). This implied that miR-367 may play different roles in various tumors. In the study of NSCLC, miR-367 was expressed higher in cancer tissues than that in normal lung tissue, and its high expression was closely related to the poor prognosis of the patients (9). However, its mechanisms in the tumorigenesis of NSCLC are still unknown.

F-box/WD-40 domain protein 7 (FBXW7), one of the best studied F-box proteins, participates in ubiquitination and degradation of target proteins, such as c-Myc, c-Jun and cyclin E (10). Most of its target proteins are oncoproteins, and low expression of FBXW7 is correlated with the poor prognosis of cancer patients; thus, it was recognized as a powerful tumor suppressor. FBXW7 takes part in multiple signaling pathways, including cell proliferation, apoptosis, migration 
and metastasis. Previous studies have shown that the expression of Fbxw7 is regulated by multiple transcription factors, among which miRNA is an important post-transcriptional regulatory factor. For instance, miR-223 (11) and miR-25 (12) promote proliferation and inhibit apoptosis in gastric cancer by directly suppressing FBXW7.

In the present study, we found that the level of miR-367 was higher in NSCLC tissues and cells than levels in normal lung tissues and cells, and its high expression was indicative of a poor patient prognosis. However, the levels of miR-367 and FBXW7 were negative correlated in NSCLC tissues. In addition, we demonstrated that miR-367 promoted cell proliferation, cell cycle progression and inhibited apoptosis by directly binding to and inhibiting FBXW7 in vivo and in vitro. Therefore, miR-367 may be a crucial target for the early diagnosis and treatment of NSCLC.

\section{Materials and methods}

Patients and clinical specimens. Sixty NSCLC patients who underwent pulmonary surgery in our department from January 2013 to January 2016 were included in the present study. None of the patients had received neoadjuvant radiotherapy/chemotherapy before surgery. All patients with NSCLC were confirmed by histopathologic evaluation. Written informed consent was obtained from the patients in accordance with the Declaration of Helsinki before sample collection. Immediately after resection, NSCLC and matched normal adjacent tissues (not less than $50 \mathrm{~mm}$ away from the NSCLC) specimens were stored at $-80^{\circ} \mathrm{C}$ until required. The present study was approved by the Ethics Committee of The First Affiliated Hospital of Xi'an Jiaotong University. Clinicopathological parameters are listed in Table I.

Cell culture and transfection. Human bronchial epithelial cells (Beas-2B) and three NSCLC cell lines (A549, H460 and H1299) were obtained from the American Type Culture Collection (ATCC; Manassas, VA, USA), while the 95D cell line was obtained from the China Center for Type Culture Collection (CCTCC; Wuhan, China). All cell lines were incubated at $37^{\circ} \mathrm{C}$ in a humidified atmosphere with $5 \% \mathrm{CO}_{2}$. Beas-2B, A549, H460 and 95D cells were grown in Dulbecco's modified Eagle's medium (DMEM) and H1299 was grown in Roswell Park Memorial Institute (RPMI)-1640 medium (both from HyClone, Logan, UT, USA), supplemented with $10 \%$ heat inactivated fetal bovine serum (FBS; Excell Bio, Shanghai, China), penicillin $(100 \mu \mathrm{g} / \mathrm{ml})$, streptomycin $(100 \mathrm{mg} / \mathrm{ml})$ solution (HyClone). For miR-367 transient transfection, cells at $50 \%$ confluence were transfected with micrON ${ }^{\mathrm{TM}}$ hsa-miR367-3p mimic, micrOFF ${ }^{\mathrm{TM}}$ hsa-miR-367-3p inhibitor (RiboBio) or corresponding non-specific ncRNA controls without homologous to any known human gene sequences (both from RiboBio, Guangzhou, China), using X-tremeGENE siRNA Transfection Reagent (Roche Diagnostics, Indianapolis, IN, USA). FBXW7-specific siRNA and control siRNA were previously reported (13).

Vector constructs. To construct the FBXW7 expression vector, the open reading frame (ORF) of the human FBXW7 gene (without the 3'-UTR) was amplified by PCR and subcloned
Table I. Correlation between the expression of miR-367 and the clinicopathological characteristics of the NSCLC patients.

\begin{tabular}{|c|c|c|c|c|}
\hline \multirow[b]{2}{*}{$\begin{array}{l}\text { Clinicopathological } \\
\text { characteristics }\end{array}$} & \multirow[b]{2}{*}{$\begin{array}{l}\text { No. of cases } \\
\quad(n=60)\end{array}$} & \multicolumn{2}{|c|}{$\begin{array}{c}\text { miR-367 } \\
\text { expression } \\
\text { level }\end{array}$} & \multirow[b]{2}{*}{ P-value } \\
\hline & & $\begin{array}{l}\text { High } \\
(\mathrm{n}=35)\end{array}$ & $\begin{array}{l}\text { Low } \\
(\mathrm{n}=25)\end{array}$ & \\
\hline Age (years) & & & & 0.124 \\
\hline$<60$ & 38 & 25 & 13 & \\
\hline$\geq 60$ & 22 & 10 & 12 & \\
\hline Sex & & & & 0.853 \\
\hline Male & 40 & 23 & 17 & \\
\hline Female & 20 & 12 & 8 & \\
\hline Tumor size $(\mathrm{cm})$ & & & & $0.003^{\mathrm{b}}$ \\
\hline$\leq 3$ & 25 & 9 & 16 & \\
\hline$>3$ & 35 & 26 & 9 & \\
\hline TNM tumor stage & & & & $0.034^{\mathrm{a}}$ \\
\hline $\mathrm{I}+\mathrm{II}$ & 35 & 16 & 17 & \\
\hline III+IV & 25 & 19 & 6 & \\
\hline Histology & & & & 0.582 \\
\hline Adenocarcinoma & 40 & 25 & 15 & \\
\hline Squamous cell & & & & \\
\hline Carcinoma & 13 & 7 & 6 & \\
\hline Other & 7 & 3 & 4 & \\
\hline $\begin{array}{l}\text { Degree of } \\
\text { differentiation }\end{array}$ & & & & $0.001^{\mathrm{b}}$ \\
\hline Well and moderate & 24 & 8 & 16 & \\
\hline Poor & 36 & 27 & 9 & \\
\hline $\begin{array}{l}\text { Lymph node } \\
\text { metastasis }\end{array}$ & & & & 0.193 \\
\hline Yes & 37 & 24 & 13 & \\
\hline No & 23 & 11 & 12 & \\
\hline Smoking history & & & & 0.430 \\
\hline Yes & 44 & 27 & 17 & \\
\hline No & 16 & 8 & 8 & \\
\hline
\end{tabular}

${ }^{\mathrm{a}} \mathrm{p}<0.05 ;{ }^{\mathrm{b}} \mathrm{p}<0.01$. NSCLC, non-small cell lung cancer; TNM, tumornode-metastasis

into pcDNA3.0 (Invitrogen, Life Technologies, Carlsbad, CA, USA). The fragment of FBXW7 3'-UTR containing the predicted or mutated miR-367-3p binding sites was subcloned into the XhoI site downstream of firefly luciferase in pGL3 control vector (Promega, Madison, WI, USA). The recombinant plasmids were renamed as FBXW7-3'-UTR-wt or FBXW7-3'-UTR-mt. Lipofectamine 3000 (Invitrogen) was used for transfection of plasmid alone or along with RNA oligonucleotides, according to the manufacturer's instructions.

Quantitative real-time PCR ( $q R T-P C R)$ and western blotting. Total RNA were extracted from NSCLC tissues or cell lines using TRIzol reagent (Invitrogen). Total mRNA was reverse-transcribed into cDNA using Mir-X miRNA qRT-PCR 
Table II. Human primer sequences used for qRT-PCR.

\begin{tabular}{lll}
\hline Primers & \multicolumn{1}{c}{ Forward (5'-3') } & \multicolumn{1}{c}{ Reverse (5'-3') } \\
\hline FBXW7 & CACTCAAAGTGTGGAATGCAGAGAC & GCATCTCGAGAACCGCTAACAA \\
U6 & CTCGCTTCGGCAGCACA & AACGCTTCACGAATTTGCGT \\
GAPDH & CGGAGTCAACGGATTTGGTCGTAT & AGCCTTCTCCATGGTGAAGAC
\end{tabular}

SYBR Kit or Universal cDNA Synthesis Kit II (Takara, Dalian, China) according to the manufacturer's instructions. With the use of a CFX96 Real-Time PCR Detection System (Bio-Rad, Hercules, CA, USA), real-time quantitative PCR (RT-qPCR) was performed in triplicate for each sample in a $25-\mu 1$ reaction volume with SYBR ${ }^{\circledR}$ Premix Ex Taq ${ }^{\mathrm{TM}}$ II (Takara). The expression of FBXW7 and miR-367 relative to endogenous control (GAPDH or U6) were calculated using the $2^{-\Delta \Delta C t}$ method. The primers specific for miR-367-3p were obtained from RiboBio, and other primer sequences are listed in Table II and synthesized by Sangon Biotech (Shanghai, China).

Protein extraction and western blot analysis were performed as previously described (14). The primary antibodies used for western blotting were as follows: FBXW7 (1:1,000; ab109617; Abcam, Cambridge, MA, USA), c-Myc (1:500; 10828-1-AP; Proteintech, Wuhan, China), c-Jun (1:500; ab32385; Abcam). $\beta$-actin (1:1,000; 20536-1-AP; Proteintech).

Cell proliferation assay. Cell proliferation of the NSCLC cell lines was evaluated using a WST-8 assay as previously described (15). After being routinely incubated for $24 \mathrm{~h}$ in different treatments, cells were seeded into 96-well plates at a density of $2 \times 10^{3}$ cells/well and cultured for another 24, 48 and $72 \mathrm{~h}$, separately. The cells were then washed with phosphate-buffered saline (PBS), and incubated in complete medium with $10 \%$ Cell Counting Kit-8 (CCK-8) solution (Beyotime, Beijing, China) at $37^{\circ} \mathrm{C}$ for $4 \mathrm{~h}$. The absorbance of each well was measured using a mircoplate reader at $450 \mathrm{~nm}$. These experiments were carried out in triplicate.

Cell cycle assay. After transfection with the plasmids or RNA oligonucleotides for $48 \mathrm{~h}$, cells were washed twice with cold PBS and fixed with $500 \mu 1$ of $70 \%$ cold ethanol at $4^{\circ} \mathrm{C}$ overnight. The cells were then incubated with $100 \mu \mathrm{l}$ RNase at $37^{\circ} \mathrm{C}$ for $30 \mathrm{~min}$ and stained with $400 \mathrm{mg} / \mathrm{ml}$ propidium iodide (PI) (KGA512; KeyGen, Nanjing, China), and analyzed using FACSCalibur flow cytometry (BD Biosciences, Franklin Lakes, NJ, USA).

Cell apoptosis assay. Cell apoptosis was assessed by flow cytometry with Annexin V FITC/PI apoptosis detection kit (BD Biosciences). Briefly, cells were collected and suspended in binding buffer, and then stained using DNA staining solution (Annexin V-FITC and PI). Subsequently, early and late apoptotic, or necrotic cells were quantified and analyzed using FACSCalibur flow cytometry.

Luciferase reporter assays. For analysis of luciferase activity, HEK293 cells were seeded at a density of $2 \times 10^{5} /$ well of 24 -well plates for $24 \mathrm{~h}$ before transfection. The cells were co-trans- fected with luciferase reporter vectors (FBXW7-3'-UTR-wt or FBXW7-3'-UTR-mt) and miR-367-3p mimics/inhibitor or non-specific ncRNA controls, using Lipofectamine 3000. Renilla luciferase plasmid (100 ng/well; Promega) was used as an internal control and co-transfected with the described vectors. Luciferase activities (firefly and Renilla) were determined $48 \mathrm{~h}$ after transfection using the Dual-Luciferase ${ }^{\circledR}$ Reporter Assay System (Promega). Each luciferase assay was performed in triplicate.

Animals and in vivo treatment. Four-week-old BALB/c athymic nude mice (bisexual each half) were obtained from the Center of Laboratory Animals of Xi'an Jiaotong University. All experimental procedures involving animals were approved by the Ethics Committee of the First Affiliated Hospital of Xi'an Jiaotong University. Antagomir-367- and antagomir-NCtreated H1299 cells $\left(1 \times 10^{7}\right)$ were suspended in $150 \mu \mathrm{l}$ PBS, and then subcutaneously inoculated into the flank of nude mouse. After two weeks, tumor volume (V) was determined by measuring tumor length (L) and width (W) once every three days with a Vernier caliper, and then calculated in accordance with the formula: $\mathrm{V}=\left(\mathrm{L} \mathrm{x} \mathrm{W}^{2}\right) \times 0.5$. At the end of the experiment, all mice were sacrificed at 30 days after cell injection, and xenograft tumors were removed and weighed. The expression of miR-367 and FBXW7 in the isolated tumor tissues was detected using qRT-PCR and western blotting.

Statistical analysis. All data were analyzed using SPSS 20 and GraphPad Prism 5. All numerical data are shown as mean \pm standard deviation (SD). Comparison between different groups was carried out using t-test or ANOVA, as appropriate. The associations between the level of miR-367 and clinicopathological parameters were evaluated using Chi-square $\left(\chi^{2}\right)$ or Fisher's exact test. Univariate survival analysis was performed using the Kaplan-Meier method and log-rank test. Pearson correlation coefficient was used to examine the correlation between miR-367 and FBXW7 mRNA. Each experiment was repeated in triplicates. $\mathrm{P}<0.05$ was considered statistically significant.

\section{Results}

miR-367 is highy expressed in NSCLC. To identify the level of miR-367 in NSCLC tissues and cells, we examined the RNA level in 60 pairs of NSCLC tissues and non-tumor adjacent tissues by qRT-PCR. The result revealed that miR-367 RNA level was significantly higher in tumor tissues compared with matched adjacent non-tumor tissues ( $<<0.001$; Fig. 1A). Similar to the observations in clinical samples, miR-367 expression was also found to be significantly higher in NSCLC 

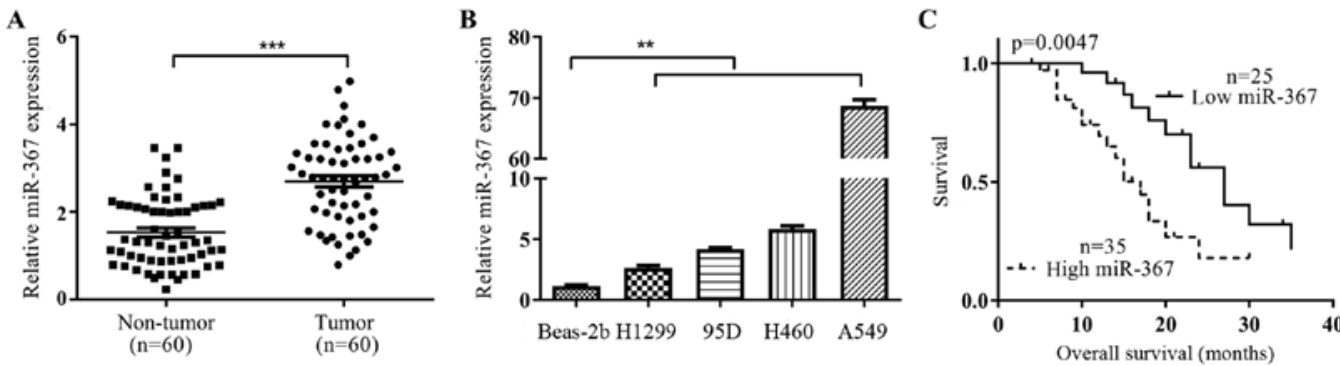

Figure 1. miR-367 is upregulated in NSCLC and is correlated with the patient prognosis. (A) qRT-PCR analysis of miR-367 expression in 60 paired tumor tissues and corresponding non-tumor adjacent tissues. The results were normalized to endogenous control (U6 RNA). Statistical significance was evaluated using Student's t-test; ${ }^{* * *}$ p $<0.001$. (B) qRT-PCR analysis of miR-367 expression in human bronchial epithelial cells and four NSCLC cell lines, using U6 as endogenous control. Data are presented as mean $\pm \mathrm{SD}\left({ }^{* *} \mathrm{p}<0.001\right.$; ANOVA test). (C) Kaplan-Meier survival analysis of NSCLC patients were plotted according to the level of miR-367 expression ( $\mathrm{p}=0.0047$; log-rank test). $\mathrm{n}=3$ replicates with similar data; statistical significance levels, ${ }^{* *} \mathrm{p}<0.01 ;{ }^{* * *} \mathrm{p}<0.001$.

A

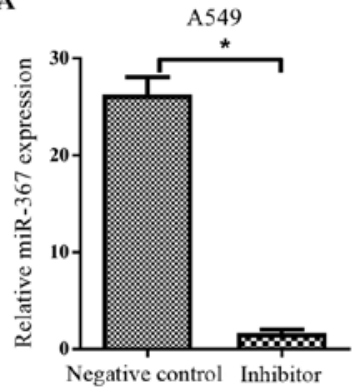

C
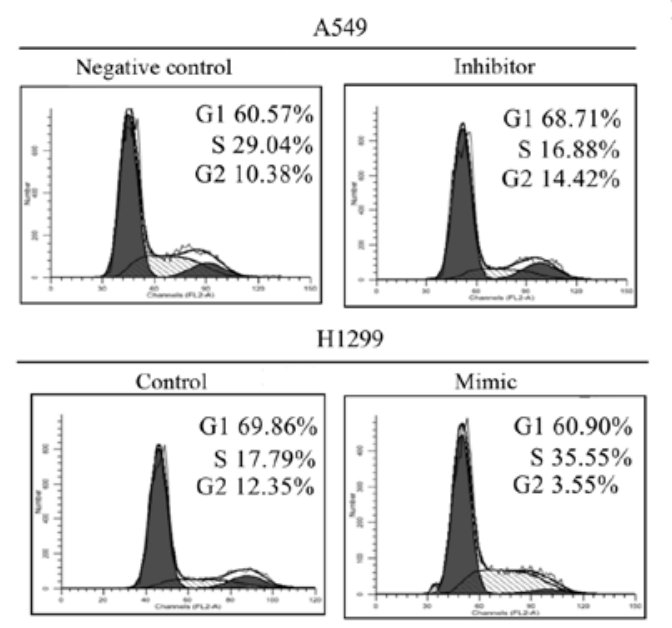

H1299
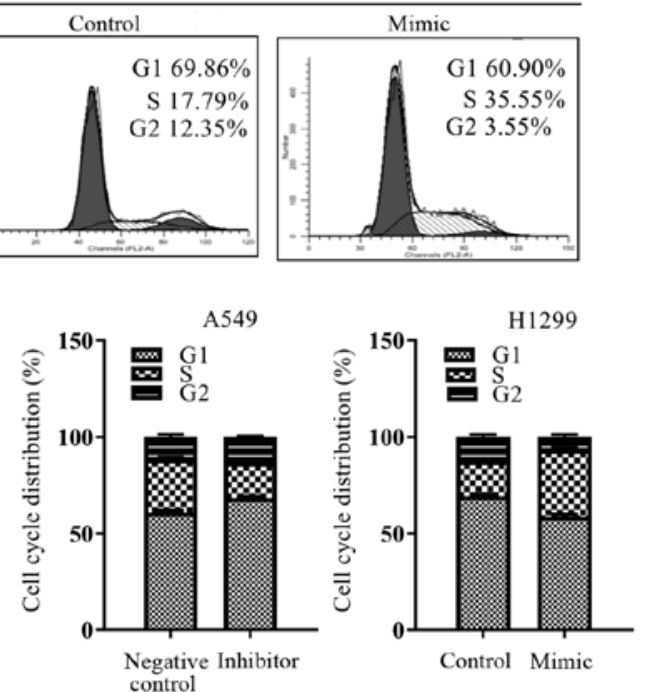

B

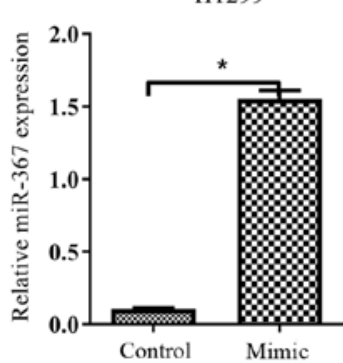

D
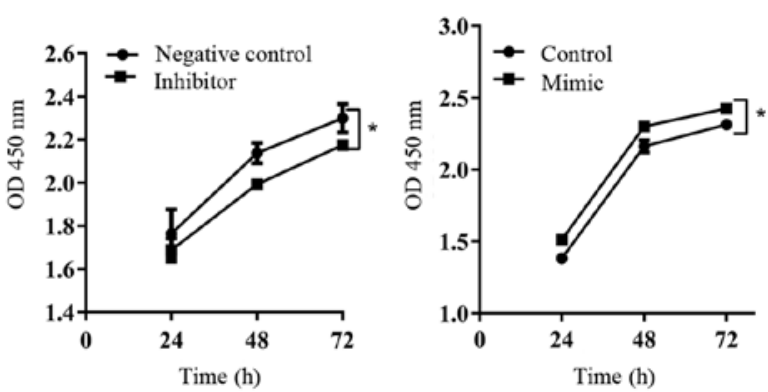

A549
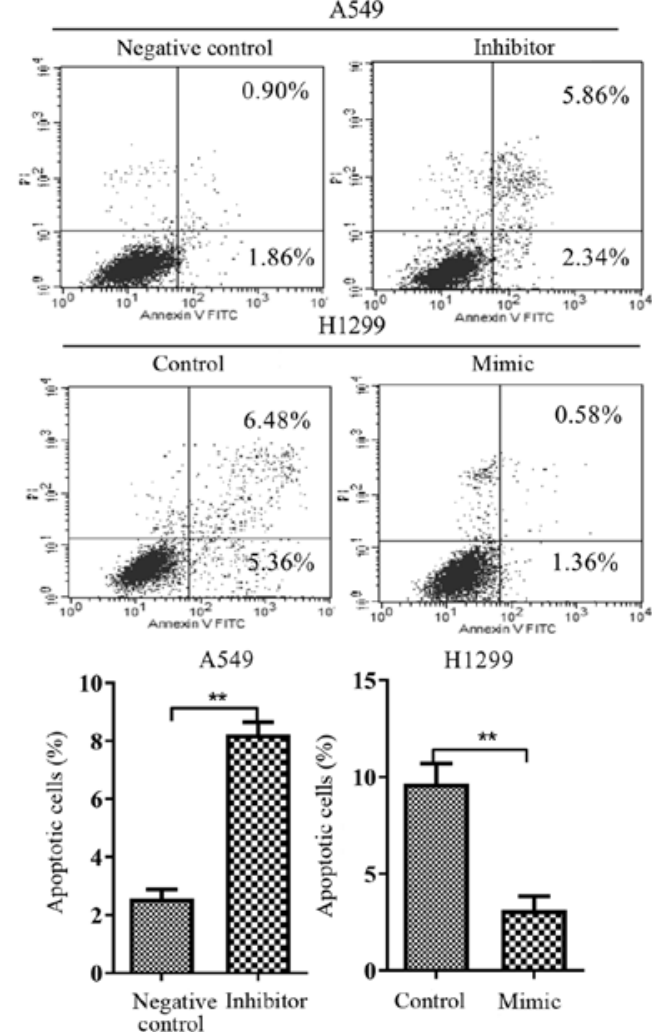

Figure 2. miR-367 promotes cell proliferation, cell cycle transition and inhibits apoptosis. (A) qRT-PCR analysis of miR-367 expression in A549 and H1299 cells after transfection with miR-367 mimics or inhibitors, respectively (" ${ }^{*}<0.05$; Student's t-test). (B) Effect of miR-367 on cell proliferation was determined by CCK-8 assay ("p $<0.05$; Student's t-test). (C) Quantification of cell cycle and (D) apoptosis as detected by flow cytometry (** $<<0.01$; both assays, ANOVA test). These experiments were repeated three times; statistical significance level, ${ }^{*} \mathrm{p}<0.05 ;{ }^{* *} \mathrm{p}<0.01$.

cell lines (A549, H1299, H460 and 95D) as compared to the normal bronchial epithelial cells Beas-2B ( $<<0.01$; Fig. 1B).
To further identify the importance of miR-367 in NSCLC progression, the correlation between miR-367 expression and 


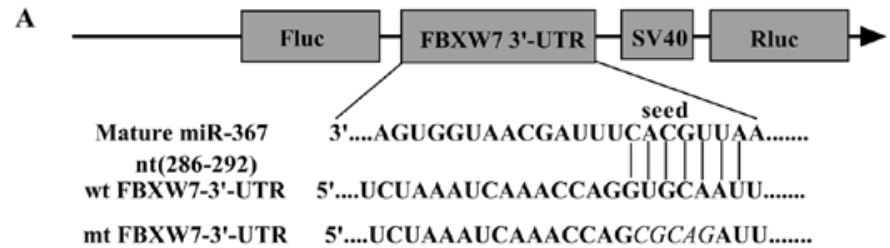

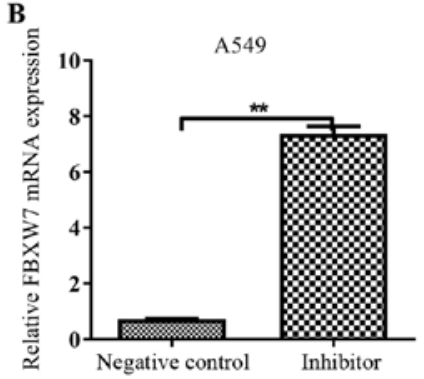

D

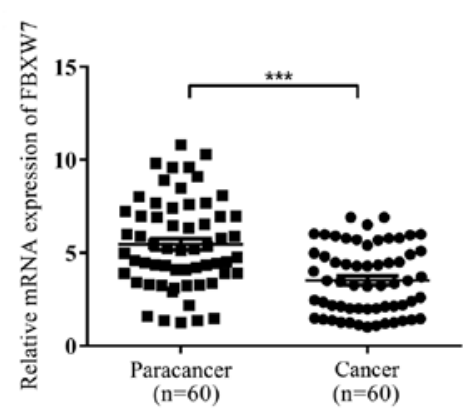

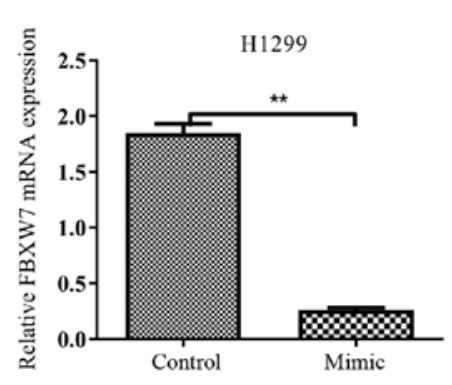

C

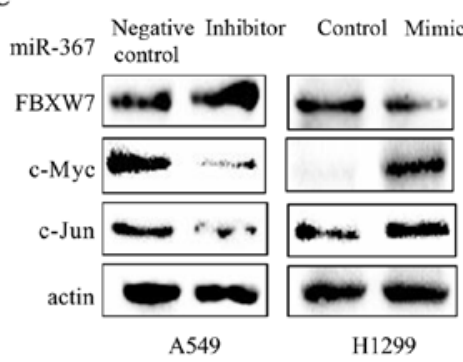

F

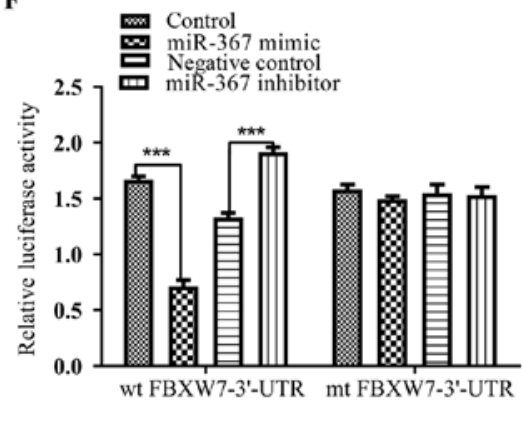

Figure 3. miR-367 directly targets FBXW7 in NSCLC. (A) A luciferase report system vector containing the wild-type or mutant miR-367-binding sequence was constructed. Mutation was generated in the complementary sequence for the seed region of miR-367. (B) The mRNA expression of FBXW7 was determinated by qRT-PCR in NSCLC cells after transfection with miR-367 mimics or inhibitors, and normalized to GAPDH ( ${ }^{* *}$ p<0.01; Student's t-test). (C) The impact of miR-367-3p mimic or inhibitor on FBXW7, c-Myc, c-Jun protein levels was compared in A549 and H1299 cells. (D) The mRNA expression of FBXW7 in NSCLC tissues was markedly lower than that corresponding non-tumor adjacent tissue $\left({ }^{* * *} \mathrm{p}<0.001\right.$; Student's $\mathrm{t}$-test). (E) The inverse correlation between miR-367 and FBXW7 mRNA expression in NSCLC tissues ( $\mathrm{r}=-0.4305$, p=0.0006; Pearson's correlation coefficient). (F) Luciferase activity was determined $48 \mathrm{~h}$ after transfection in HEK293 cells. The expression of miR-367 alteration significantly affected relative luciferase activities in HEK293 cells transfected with pGL3-FBXW7-3'-UTR-wt, but not in HEK293 cells transfected with pGL3-FBXW7-3'-UTR-mt (wt, wide type; mt mutant type) ('* Data are represented as mean $\pm \mathrm{SD}, \mathrm{n}=3$ independent experiments; statistical significance level, ${ }^{* *} \mathrm{p}<0.01 ;{ }^{* * * *} \mathrm{p}<0.001$.

clinicopathological characteristics was analyzed. As shown in Table I, high expression of miR-367 was positively associated with tumor-node-metastasis (TNM) $(\mathrm{p}=0.034)$, degree of differentiation $(p=0.001)$, tumor size $(p=0.003)$. Moreover, we investigated the prognostic value of miR-367. NSCLC patients with high expression of miR-367 had shorter overall survival (OS) ( $\mathrm{p}=0.0047$; Fig. 1C) using Kaplen-Merier analysis. Therefore, miR-367 may be a very important prognostic indicator in NSCLC.

Effect of miR-367 on proliferation, cell cycle distribution and apoptosis in NSCLC cells. Overexpression or loss of miRNA is normally associated with the change in biological functions. We ectopically expressed miR-367 using miR-367-3p mimics in the H1299 cell line and inhibited endogenous miR-367 activity through miR-367-3p inhibitors in the A549 cell line. After transfection, the expression of miR-367 was significantly changed in the A549 and H1299 cells (p<0.01; Fig. 2A). Next, we examined the effects of miR-367 on cell proliferation, cell cycle and apoptosis. CCK-8 assays demonstrated a statistically significant increase in proliferation after miR-367 upregula- tion, whereas downregulation of miR-367 in A549 cells showed a significant decrease in cell proliferation as compared to the control cells ( $\mathrm{p}<0.01$; Fig. 2B). Furthermore, as determined by flow cytometric analysis, miR-367 overexpression significantly decreased the cellular population of the $\mathrm{G} 0 / \mathrm{G} 1$ phase and led to a significant increase in $\mathrm{S}$ phase cells ( $\mathrm{p}<0.05$; Fig. $2 \mathrm{C}$ ), while its downregulation had the opposite result ( $<<0.05$; Fig. $2 \mathrm{C}$ ). Moreover, apoptosis assay determined by flow cytometry, presented that miR-367 overexpression prominently reduced the apoptosis ratio of H1299 cells ( $<<0.05$; Fig. 2D), but the inhibition of miR-367 markedly increased the percentage of apoptotic A549 cells (p<0.05; Fig. 2D). Taken together, these data suggest an essential role for miR-367 as a mediator of the biological effects of NSCLC cells.

miR-367 negatively regulates FBXW7 in NSCLC cells. To ascertain which potential targets are responsible for the biological functions of miR-367 in NSCLC cells, we screened for potential targets of miR-367-3p using three miRNA target prediction programs (miRanda, TargetScan and PicTar). Eventually, we predicted that FBXW7 may be 
A

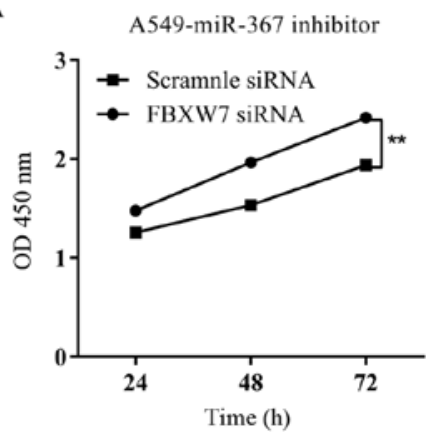

H1299-miR-367 mimic

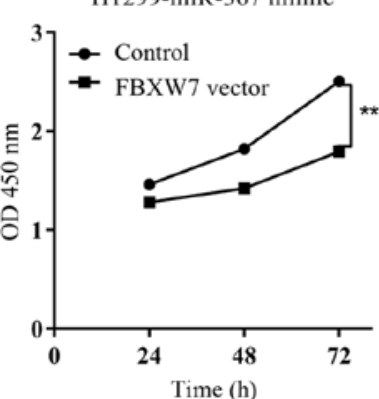

B

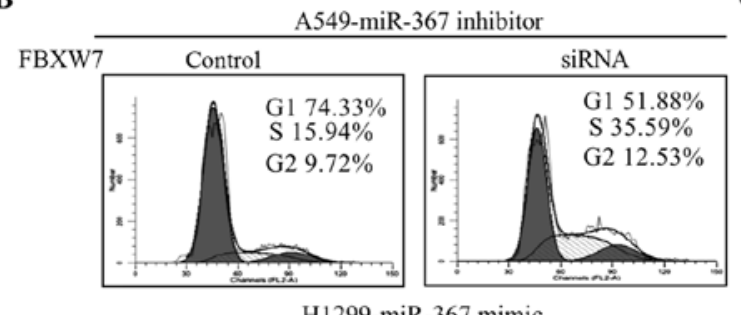

H1299-miR-367 mimic

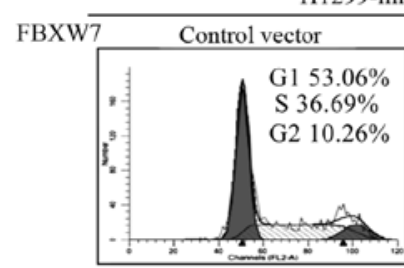

A549-miR-367 inhibitor

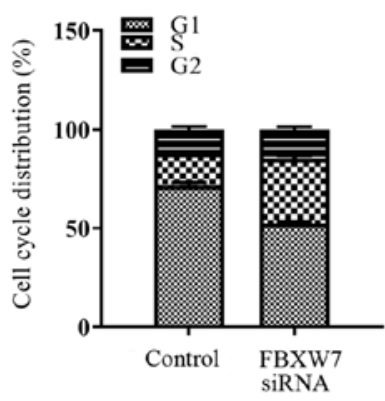

H1299-miR-367mimic

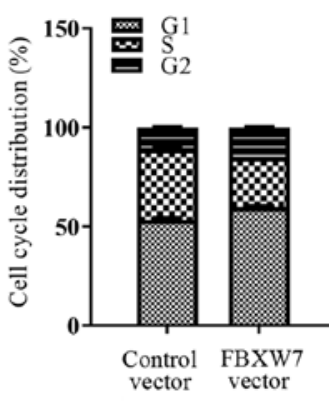

C
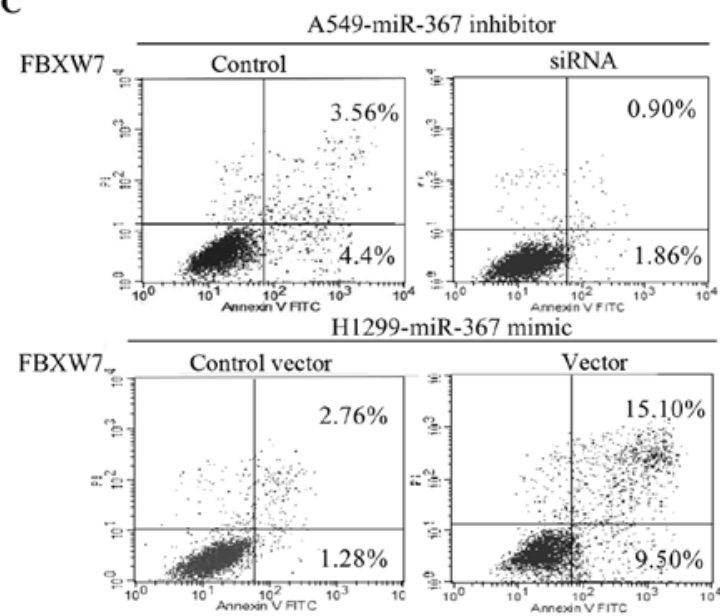

A549-miR-367 inhibitor
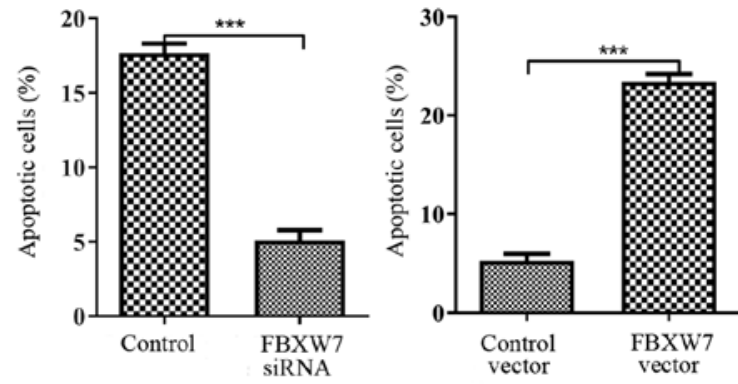

D

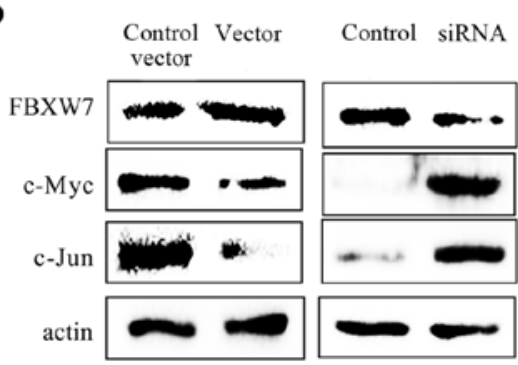

H1299-miR-367 mimic A549-miR-367 inhibitor

Figure 4. FBXW7 reverses miR-367-mediated biological function in NSCLC cell lines. (A-C) H1299 cells was co-transfected with FBXW7 expression plasmid or empty vector (EV) and miR-367 mimics, while A549 cells was co-transfected with scrambled siRNA or FBXW7 siRNA and miR-367 inhibitors After that, all transfected cells were collected and analyzed for (A) cell proliferation, (B) cell cycle and (C) apoptosis. (D) The expression changes in FBXW7 abrogate the impact of miR-367 on the protein expression of c-Myc and c-Jun. $n=3$ independent experiments. Statistical significance level, ${ }^{* *}$ p $<0.01 ;{ }^{* * *}$ p $<0.001$ as determined using Student's t-test.

a target of miR-367, as 3'-UTR of FBXW7 mRNA harbors a highly conserved binding site that is complementary to the miR-367 seed sequence (Fig. 3A). In order to validate the role of miR-367 in FBXW7 expression regulation, we detected the mRNA expression of FBXW7 in NSCLC cells transfected with miR-367 mimics or inhibitors, respectively. 
A

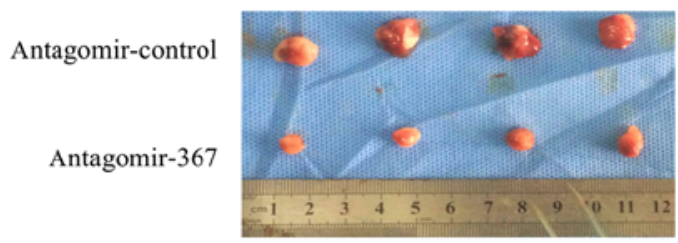

D

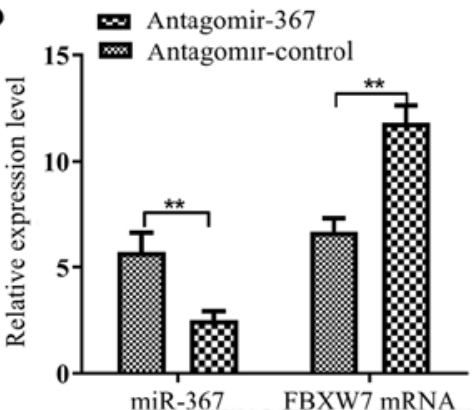

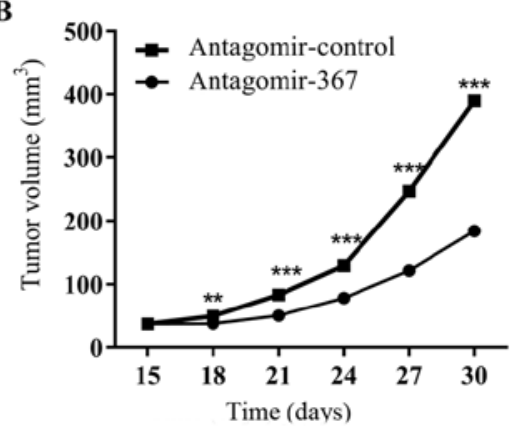

E

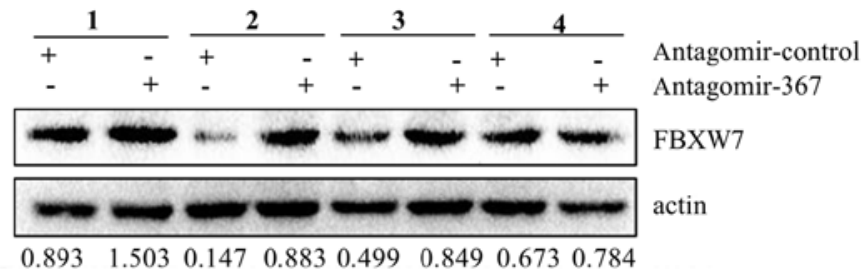

Figure 5. miR-367 promotes NSCLC progression in vivo. (A) The gross morphology of tumors (upper panel, NC group; lower panel, ant-miR-367 group). (B) Tumor growth curve and (C) average tumor weight showed that tumor formation was suppressed by agomir-367 treatment in the H1299 cell xenograft model. The growth of xenografts was observed for 4 weeks. (D) The mRNA expression of miR-367 and FBXW7 in tumor tissues from the animals. (E) The protein levels of FBXW7 in the tumor tissues from the tumor samples of nude mice. These data were analyzed by ANOVA or t-test, statistical significance level, ${ }^{* *} \mathrm{p}<0.01 ;{ }^{* * *} \mathrm{p}<0.001$.

miR-367 mimic significantly inhibited the mRNA and protein levels of FBXW7 in $\mathrm{H} 1299$ cells $(\mathrm{p}<0.05$; respectively, Fig. 3B and C), while inhibition of endogenous miR-367 using a synthetic miR-367 inhibitor significantly enhanced FBXW7 expression in A549 cells ( $\mathrm{p}<0.05$; respectively, Fig. 3B and C). In addition, we also detected the protein expression of c-Myc and c-Jun, which could be directly downregulated by FBXW7. In contrast to FBXW7, the protein expression of c-Myc and c-Jun were reversely changed after miR-367 mimic or inhibitor treatment $(\mathrm{p}<0.05$; Fig. $3 \mathrm{C})$. To further analyze the correlation between miR-367 and FBXW7 in NSCLC tissues. We examined mRNA levels of FBXW7 in 60 paired clinical NSCLC and non-cancerous tissues. Our data revealed that the mRNA expression levels of FBXW7 were decreased in NSCLC tissues compared with the adjacent normal tissues $(\mathrm{p}<0.05$; Fig. 3D). Markedly, The Pearson's correlation analysis showed that miR-367 was inversely correlated with FBXW7 expression in 60 samples ( $r=-0.4305, p=0.0006)$ (Fig. 3E). We then utilized a dual-luciferase reporter system to determine whether miR-367-3p effectively targets the 3'-UTR of FBXW7. Luciferase reporter assays revealed that ectopic expression of miR-367-3p mimics inhibited the luciferase activity of FBXW7-3'-UTR-wt reporter in HEK-293, whereas miR-367 knockdown significantly increased the luciferase activity of FBXW7-3'-UTR-wt reporter ( $<<0.001$; Fig. 3F). In contrast, no significant luciferase alteration was observed in cells transfected with mutant vector. These results strongly indicated that FBXW7 is a direct target of miR-367-3p.

FBXW7 partly reverses the effect of miR-367 on NSCLC cells. Most of the biological effects of miRNAs are altered through regulation of their target genes. Thus, we assessed the influence of FBXW7 alteration for the biological function of miR-367. Then, we knocked down or upregulated the expression of FBXW7 using siRNA or pcDNA3.0-FBXW7, respectively. As expected, FBXW7 expression plasmid efficiently abrogated the cell proliferation, cell cycle acceleration and apoptosis inhibition induced by miR-367 mimic in H1299 cells ( $\mathrm{p}<0.01$; respectively, Fig. 4A-C). Meanwhile, the effects of miR-367 inhibitor on cell proliferation, cell cycle and apoptosis were attenuated by siFBXW7 in the A549 cells ( $<<0.01$; respectively, Fig. 4A-C). Finally, restoration of FBXW7 expression suppressed c-Myc and c-Jun expression (Fig. 4D). Conversely, FBXW7 silencing increased the expression of c-Myc and c-Jun (Fig. 4D). Taken together, these results demonstrated that FBXW7 mediated the carcinogenesis properties of miR-367 in NSCLC cells.

miR-367 promotes NSCLC tumor growth in vivo. Considering the important roles of miR-367 in NSCLC, the potential therapeutic effects of miR-367 attracted our attention. Tumor growth of xenografts was significantly inhibited in the group treated with antagomir-367 $(\mathrm{p}<0.001$; Fig. 5A) compared to the negative control group. This trend was also verified by the sizes $(\mathrm{p}<0.0001$; Fig. 5B) and weights $(\mathrm{p}<0.001$; Fig. 5C) of the tumors excised from the nude mice. qRT-PCR and western blot analysis further revealed that the mRNA and protein expression levels of FBXW7 were significantly increased in the antagomir-367-treated xenograft $(\mathrm{p}<0.05$; respectively, Fig. 5D and E) when the miR-367 level was decreased. These data indicated that miR-367 was capable of promoting tumor growth and inhibiting FBXW7 expression in vivo. 


\section{Discussion}

Dysregulation of miR-367 has been found in several types of human malignant tumors, including osteosarcoma (16), glioma (17), breast (18) and esophageal cancer (19). High expression of miR-367 in most of these tumor tissues was closely related to the poor prognosis of patients. A recent study also reported that the high expression of miR-367 predicted poor prognosis in patients with NSCLC (9). However, the present study only evaluated the clinical significance of miR-367 in NSCLC, but did not explore the mechanisms of miR-367 in vitro and in vivo. In the present study, similar results were observed in clinical specimen analysis. However, we also found that high miR-367 expression was positively correlated with TNM, degree of differentiation, tumor size and poorer prognosis of NSCLC patients. Consistent with the results in clinical NSCLC samples, miR-367 expression was significantly higher in several NSCLC cell lines compared with human bronchial epithelial cells (BEAS-2B). These results suggested that miR-367 is closely related to the malignant progression of NSCLC.

Maintaining proliferation and resisting apoptosis are two prominent hallmarks of cancer cells (20). Previous studies reported that miR-367 plays a significant role in regulating tumor cell proliferation and apoptosis. For example, miR-367 promoted the proliferation of HCC cells by inhibiting PTEN expression (5), and suppressed adriamycin-induced apoptosis of osteosarcoma cells by regulating KLF-4 (16). In addition, the downregulation of miR-367 significantly inhibited cell proliferation and cell cycle progression of esophageal cancer cells (19). However, how miR-367 regulates NSCLC cell growth remains unclear. In the present study, we showed that the reduced expression of miR-367 significantly inhibited the cell proliferation, delayed cell cycle progression and promoted apoptosis in NSCLC. To further elucidate the specific mechanism of miR-367 in NSCLC, we discovered that FBXW7 is a potential target of miR-367 using microRNA (miRNA) public databases. Several studies have also demonstrated that various miRNAs play a role in promoting proliferation and anti-apoptosis by targeting FBXW7. miR-92 promoted HCC cell proliferation and inhibited cell apoptosis by targeting FBXW7 (21). miR-223 contributed to apoptosis reduction and increased proliferation and invasion in gastric cancer cell lines by inhibiting FBXW7 (11). In the present study, our results firstly confirmed that miR-367-3p directly bound to and regulated the function of FBXW7. Supportively, a significantly inverse correlation was found between miR-367 and FBXW7 mRNA expression in NSCLC tissues. Moreover, we also investigated the effect of FBXW7 on the biological role of miR-367 and finally found that FBXW7 reversed miR-367-induced cell proliferation, cell cycle and apoptosis inhibition. Our findings, therefore, provide a mechanism that miR-367 may promote cell growth by regulating FBXW7 in NSCLC.

Much evidence has indicated that the tumor suppressor function of FBXW7 is mainly regulated by ubiquitin-mediated degradation of several oncoproteins. Downregulation of FBXW7 promoted the proliferation of colon cancer cells by increasing the expression of c-Myc and cyclin E (22). Conversely, upregulation of FBXW7 in renal carcinoma cell inhibited cell proliferation and promoted apoptosis by suppressing c-Myc and c-Jun expression (23). c-Myc and c-Jun, as the direct targets of FBXW7, are important oncoproteins related to proliferation and apoptosis in many malignant tumors. c-Myc can directly activate cyclin E-cdk2 complex, mediate phosphorylation of Rb, and thereby activate E2F transcription regulation factor to promote DNA replication (24). The phosphorylation of c-Jun, another target protein of FBXW7, participates in promoting the proliferation and cell cycle by activating cyclin D (25). In the present study, miR-367 mimic/inhibitor may decrease/increase the expression of FBXW7 by changing the level of miR-367, and then increase/decrease the expression of c-Jun and c-Myc in NSCLC cells. In addition, overexpression/knockdown of FBXW7 may reverse the function of miR-367 by decreasing/increasing the expression of c-Myc and c-Jun under the treatment of miR-367 mimic/inhibitor. Given that c-Myc and c-Jun are known as growth promoters whose abnormal expression may induce tumorigenesis, we speculated that the miR-367/FBXW7/c-Myc and miR-367/FBXW7/c-Jun signaling pathway may play pivotal roles in the development of NSCLC carcinogenesis.

Nevertheless, the present study focused on the effect of miR-367 on the basic biological functions of NSCLC cells. As an important regulatory factor involved in self-renewal and pluripotency of embryonic stem cells, miR-367 also plays a vital role in regulating the function of cancer stem cells (6). miR-367 overexpression significantly promoted stem-like traits in medulloblastoma cell lines. Meanwhile, stem cellspecific transcription factor Oct 4 promoted the expression of miR-367. Upregulation of miR-367 also increased the expression of Oct 4 or Sox 2 in medulloblastoma cell lines. As is known to all, the miR-302/367 family and special transcription factors (Oct4, Sox 2 and Nanog) have a positive feedback loop of self-regulation $(26,27)$. Notably, FBXW7 was found to negatively regulated the protein expression of Sox 2 and Nanog in cholangiocarcinoma (28) and colorectal cancer cells (29). Thus, we may further explore the role of miR-367 and FBXW7 in the regulation of NSCLC stem cells in future research.

In conclusion, we demonstrated that miR-367 was expressed at a higher level in NSCLC tissues and cells than levels in normal lung tissues and cells, and its high expression was statistically correlated with the poor prognosis of NSCLC patients. Moreover, miR-367 and FBXW7 mRNA expression in NSCLC tissues was negatively correlated. The experiments in vivo and in vitro showed that miR-367 promoted the cell proliferation, cell cycle progression and inhibited cell apoptosis in NSCLC cells by negatively regulating FBXW7 expression. Hence, miR-367 may serve as a novel target for the diagnosis and treatment of NSCLC.

\section{Acknowledgements}

The present study was supported by grants from the National Natural Science Foundation of China (no. 81272418) (to H.R).

\section{References}

1. Torre LA, Bray F, Siegel RL, Ferlay J, Lortet-Tieulent J and Jemal A: Global cancer statistics, 2012. CA Cancer J Clin 65: 87-108, 2015

2. Taylor MD, Nagji AS, Bhamidipati CM, Theodosakis $\mathrm{N}$, Kozower BD, Lau CL and Jones DR: Tumor recurrence after complete resection for non-small cell lung cancer. Ann Thorac Surg 93: 1813-1821, 2012. 
3. Gao Z, Zhu X and Dou Y: The miR-302/367 cluster: A comprehensive update on its evolution and functions. Open Biol 5: 150138, 2015.

4. Barroso-del Jesus A, Lucena-Aguilar G and Menendez P: The miR-302-367 cluster as a potential stemness regulator in ESCs. Cell Cycle 8: 394-398, 2009.

5. Meng X, Lu P and Fan Q: miR-367 promotes proliferation and invasion of hepatocellular carcinoma cells by negatively regulating PTEN. Biochem Biophys Res Commun 470: 187-191, 2016.

6. Kaid C, Silva PBG, Cortez BA, Rodini CO, Semedo-Kuriki P and Okamoto OK: miR-367 promotes proliferation and stem-like traits in medulloblastoma cells. Cancer Sci 106: 1188-1195, 2015

7. Zhu Z, Xu Y, Zhao J, Liu Q, Feng W, Fan J and Wang P: miR-367 promotes epithelial-to-mesenchymal transition and invasion of pancreatic ductal adenocarcinoma cells by targeting the Smad7-TGF- $\beta$ signalling pathway. Br J Cancer 112: 1367-1375, 2015.

8. Bin Z, Dedong H, Xiangjie F, Hongwei X and Qinghui Y: The microRNA-367 inhibits the invasion and metastasis of gastric cancer by directly repressing Rab23. Genet Test Mol Biomarkers 19: 69-74, 2015.

9. Campayo M, Navarro A, Viñolas N, Diaz T, Tejero R, Gimferrer JM, Molins L, Cabanas ML, Ramirez J, Monzo M, et al: Low miR-145 and high miR-367 are associated with unfavourable prognosis in resected nonsmall cell lung cancer. Eur Respir J 41: 1172-1178, 2013.

10. Akhoondi S, Sun D, von der Lehr N, Apostolidou S, Klotz K, Maljukova A, Cepeda D, Fiegl H, Dafou D, Marth C, et al: $F B X W 7 / h C D C 4$ is a general tumor suppressor in human cancer. Cancer Res 67: 9006-9012, 2007.

11. Li J, Guo Y, Liang X, Sun M, Wang G, De W and Wu W: MicroRNA-223 functions as an oncogene in human gastric cancer by targeting $\mathrm{FBXW} 7 / \mathrm{hCdc} 4$. J Cancer Res Clin Oncol 138: 763-774, 2012.

12. Gong J, Cui Z, Li L, Ma Q, Wang Q, Gao Y and Sun H: MicroRNA-25 promotes gastric cancer proliferation, invasion, and migration by directly targeting F-box and WD-40 Domain Protein 7, FBXW7. Tumour Biol 36: 7831-7840, 2015.

13. Ren H, Koo J, Guan B, Yue P, Deng X, Chen M, Khuri FR and Sun SY: The E3 ubiquitin ligases $\beta$-TrCP and FBXW7 cooperatively mediates GSK3-dependent Mcl-1 degradation induced by the Akt inhibitor API-1, resulting in apoptosis. Mol Cancer 12: 146, 2013.

14. Sun X, Tang SC, Xu C, Wang C, Qin S, Du N, Liu J, Zhang Y, Li X, Luo G, et al: DICER1 regulated let-7 expression levels in p53-induced cancer repression requires cyclin D1. J Cell Mol Med 19: 1357-1365, 2015.

15. Qiang BU and Jiang H: Chemosensitivity testing for human prostatic cancer with primary culture cells and purified culture cells using the CCK-8 assay. Pharmaceutical and Clinical Research, 2013.
16. Wang GC, He QY, Tong DK, Wang CF, Liu K, Ding C, Ji F and Zhang H: MiR-367 negatively regulates apoptosis induced by adriamycin in osteosarcoma cells by targeting KLF4. J Bone Oncol 5: 51-56, 2016.

17. Guan Y, Chen L, Bao Y, Qiu B, Pang C, Cui R and Wang Y: High miR-196a and low miR-367 cooperatively correlate with unfavorable prognosis of high-grade glioma. Int J Clin Exp Pathol 8: 6576-6588, 2015

18. Zhang L, Liu Y, Song F, Zheng H, Hu L, Lu H, Liu P, Hao X, Zhang W and Chen K: Functional SNP in the microRNA-367 binding site in the 3'UTR of the calcium channel ryanodine receptor gene 3 (RYR3) affects breast cancer risk and calcification. Proc Natl Acad Sci USA 108: 13653-13658, 2011.

19. Sun J, Song K, Feng X and Gao S: MicroRNA-367 is a potential diagnostic biomarker for patients with esophageal squamous cell carcinoma. Biochem Biophys Res Commun 473: 363-369, 2016.

20. Hanahan D and Awada A: The Hallmarks of Cancer Review. Ann Oncol 100: 57-70, 2012.

21. Yang W, Dou C, Wang Y, Jia Y, Li C, Zheng X and Tu K: MicroRNA-92a contributes to tumor growth of human hepatocellular carcinoma by targeting FBXW7. Oncol Rep 34: 2576-2584, 2015.

22. Guo Z, Zhou Y, Evers BM and Wang Q: Rictor regulates FBXW7-dependent c-Myc and cyclin E degradation in colorectal cancer cells. Biochem Biophys Res Commun 418: 426-432, 2012.

23. Fu Y, Lin Y, Yang Z, Yang G, Li G, Liu Y, Tan X, Huang Y, Wu X, Wang Y, et al: FBXW7 overexpression suppresses renal cancer cell proliferation and induces apoptosis. Med Oncol 32: $215,2015$.

24. Chen J: Mechanism of molecular regulation of cyclin E-CDK2. Journal of Medical Molecular Biology, 2006.

25. Schwabe RF, Bradham CA, Uehara T, Hatano E, Bennett BL, Schoonhoven R and Brenner DA: c-Jun-N-terminal kinase drives cyclin D1 expression and proliferation during liver regeneration. Hepatology 37: 824-832, 2003.

26. Anokye-Danso F, Trivedi CM, Juhr D, Gupta M, Cui Z, Tian Y, Zhang Y, Yang W, Gruber PJ, Epstein JA, et al: Highly efficient miRNA-mediated reprogramming of mouse and human somatic cells to pluripotency. Cell Stem Cell 8: 376-388, 2011.

27. Lin SL, Chang DC, Lin CH, Ying SY, Leu D and Wu DT: Regulation of somatic cell reprogramming through inducible mir-302 expression. Nucleic Acids Res 39: 1054-1065, 2011.

28. Yang H, Lu X, Liu Z, Chen L, Xu Y, Wang Y, Wei G and Chen Y: FBXW7 suppresses epithelial-mesenchymal transition, stemness and metastatic potential of cholangiocarcinoma cells. Oncotarget 6: 6310-6325, 2015.

29. Wang Y, Liu Y, Lu J, Zhang P, Wang Y, Xu Y, Wang Z, Mao JH and Wei G: Rapamycin inhibits FBXW7 loss-induced epithelialmesenchymal transition and cancer stem cell-like characteristics in colorectal cancer cells. Biochem Biophys Res Commun 434: 352-356, 2013. 